\title{
ORDERED GAN/INGAN NANORODS ARRAYS GROWN BY MOLECULAR BEAM EPITAXY FOR PHOSPHOR-FREE WHITE LIGHT EMISSION
}

\author{
S. ALBERT ${ }^{*}$, A. BENGOECHEA-ENCABO ${ }^{*}$, M.A. SANCHEZ-GARCÍA, F. BARBAGINI, E. CALLEJA ${ }^{\dagger}$
}

E. LUNA, A. TRAMPERT, U. JAHN

P. LEFEBVRE

L.L. LÓPEZ, S. ESTRADÉ, J.M. REBLED, F. PEIRÓ

G. NATAF, P. DE MIERRY, J. ZUÑIGA-PÉREZ

\begin{abstract}
The basics of the self-assembled growth of GaN nanorods on $\mathrm{Si}(111)$ are reviewed. Morphology differences and optical properties are compared to those of GaN layers grown directly on $\mathrm{Si}(111)$. The effects of the growth temperature on the In incorporation in self-assembled InGaN nanorods grown on $\mathrm{Si}(111)$ is described. In addition, the inclusion of InGaN quantum disk structures into selfassembled GaN nanorods show clear confinement effects as a function of the quantum disk thickness. In order to overcome the properties dispersion and the intrinsic inhomogeneous nature of the self-assembled growth, the selective area growth of GaN nanorods on both, c-plane and a-plane $\mathrm{GaN}$ on sapphire templates, is addressed, with special emphasis on optical quality and morphology differences. The analysis of the optical emission from a single InGaN quantum disk is shown for both polar and non-polar nanorod orientations.
\end{abstract}

Keywords: Nanorods; GaN; InGaN; SAG; white light.

\section{Introduction}

Group III-nitride semiconductors are very appealing for applications in optoelectronic devices, in particular, InGaN alloys for light emitters working in the whole visible spectral region, ${ }^{1,2,3}$ that open the way to develop phosphor-free white light emitters.

Most research efforts focused mainly on c-plane quantum well (QW) structures, though their efficiency suffers from (i) a reduced radiative recombination rate due to the quantum confined Stark effect derived from spontaneous and piezoelectric polarizations, 
and (ii) the high density of nonradiative defects due to the lattice mismatch between GaN and InGaN.

It has been shown that dislocation- and strain- free group-III nitrides can be grown on $\mathrm{Si}(100)$ and (111), as well as on amorphous $\mathrm{SiO}_{2}$ substrates ${ }^{4,5,6,7}$ in the form of onedimensional structures such as nanorods (NRs). A potential benefit of NRs as light emitters, aside from their very high crystal quality, relies on better light extraction efficiency as compared to thin films, because of a guiding effect along the NR axis. When embedding quantum disks (QDisc) in NRs, light emission enhancement is expected due to carrier confinement. ${ }^{9,10,11,12,13}$ In addition, it has been demonstrated that white light emission can be obtained from self-assembled InGaN/GaN NRs. ${ }^{14,15,16,17}$

Self assembled arrays of InGaN/GaN nanocolumnar Light Emitting Diodes (LEDs) always show polychromatic emission mainly due to an inhomogeneous distribution of NRs geometry (diameter, height), In content, and strain distribution within the active region (QDisc). ${ }^{15,16,18}$ In addition, self-assembled NRs may merge generating extended defects as dislocations and stacking faults. Thus, efficient white LEDs based on IIInitrides grown by a self-assembled method have little chances to become commercial. However, self-assembled III-nitride NRs played a significant role in helping to understand material properties and the physics involved in efficient light emission from almost defect-free nanocrystals.

The first part of this work will address the growth of self-assembled GaN NRs on $\mathrm{Si}(111)$ substrates, as well as InGaN/GaN NRs and InGaN QDiscs embedded into GaN NRs. The second part of the work will address the selective area growth (SAG) of these structures leading to ordered arrays as functional blocs to develop actual devices (LEDs). During the last years the SAG of GaN NRs ${ }^{19,20,21,22}$ has been studied by a number of groups, allowing the fabrication of NRs with well controlled position and diameter. In this work, SAG of GaN NRs on polar (c-plane) and non-polar (a-plane) sapphire/GaN templates will be addressed. The incorporation and properties of InGaN QDiscs to both polar and non-polar NRs will be studied.

\section{Experimental}

The plasma assisted molecular beam epitaxy (PAMBE) system used in this work was equipped with a rf-plasma source providing active nitrogen and standard Knudsen cells for Ga and In. All samples were grown, either on Si(111) for self-assembled GaN NRs, or on titanium nanohole masks deposited on c-plane or a-plane GaN/sapphire templates for SAG of GaN NRs. Metal and nitrogen fluxes were calibrated in (0001) $\mathrm{GaN}$ and (0001) InN growth rate units $(\mathrm{nm} / \mathrm{min}){ }^{23}$ In wurtzite GaN and $\mathrm{InN}$ the areal densities referring to 1 monolayer (ML) are $1.14 \times 10^{15} \mathrm{GaN} / \mathrm{cm}^{2}$ and $9.17 \times 10^{14} \mathrm{InN} / \mathrm{cm}^{2}$ respectively. ${ }^{24,25}$ Once grown, the samples were studied with scanning electron microscopy (SEM), photoluminescence (PL), and transmission electron microscopy (TEM). PL experiments were carried out using a He-Cd laser with a constant power of $30 \mathrm{~mW}$ as excitation source. Due to the chromatic collection system used, different signal optimizations in the case of a broad spectrum are possible, thus potentially deforming the 
overall aspect of the spectrum. Then, the collected optical signal was systematically optimized by setting the spectrometer at the center of the visible spectrum, therefore slightly detrimental to contributions from the red and blue-violet regions.

\section{Results and Discussion}

\subsection{Growth of self-assembled nanocolumns on Si(111) substrates}

In the last years many reports on the growth of self-assembled III-nitride NRs have been published..$^{26,27,28,29,30,31}$ The basic principles that govern the self-assembled growth process may now be summarized in a brief way yielding the necessary information to understand them. This will allow later a straight comparison with SAG.

Self-assembled GaN NR grown on Si(111) have excellent structural and optical properties, as compared to those of thin films. The reason for that relies on the absence of extended defects in the NRs that are also intrinsically free of strain because of their small footprint and large surface to volume ratio. This allows NRs to "disconnect" from the substrate after a few MLs by generating dislocations at the interface. ${ }^{32}$ The selfassembled growth of GaN NRs is governed by two main factors: (i) the growth temperature and (ii) the $\mathrm{Ga} / \mathrm{N}$ ratio. The impact of these parameters on the growth has been studied extensively. ${ }^{24}$

The actual $\mathrm{Ga} / \mathrm{N}$ ratio for the growth of self-assembled NRs has to be smaller than 1 in order to prevent the coalescence (enhanced lateral growth) of the isolated $\mathrm{GaN}$ islands formed at the very beginning of the growth. Note that the actual $\mathrm{Ga} / \mathrm{N}$ ratio may substantially differ from the nominal one, because of Ga desorption and GaN decomposition that may have a strong effect depending on the temperatures used. ${ }^{23,33}$

Assuming constant Ga and $\mathrm{N}$ fluxes of $\Phi_{\mathrm{Ga}}=1 \mathrm{~nm} / \mathrm{min}$ and $\Phi_{\mathrm{N}}=6 \mathrm{~nm} / \mathrm{min}$ that yield a III/V ratio smaller than 1, lower and upper values of the growth temperature allowing the NRs growth (NR regime) can be defined. The lower temperature limit is determined by the transition from self-assembled NR regime to compact layers, at around $760^{\circ} \mathrm{C}$ (for the established fluxes), that can be understood in terms of Ga diffusion which must be high enough in order to allow for the nucleation of well separated GaN islands. ${ }^{30}$ The upper temperature limit is determined by the balance between the nominal growth rate and the Ga desorption and GaN decomposition rates, both being exponential functions of the temperature. Beyond this limit no growth is possible. ${ }^{33}$ Note that this boundary, that occurs around $810^{\circ} \mathrm{C}$ for the established fluxes, shifts towards higher temperatures when increasing the Ga flux.

Figure 1 shows SEM images of GaN samples grown directly on $\mathrm{Si}(111)$ at different temperatures and $\mathrm{Ga} / \mathrm{N}$ ratios. Figure la refers to a $\mathrm{GaN}$ layer of around $700 \mathrm{~nm}$ thickness grown at $750^{\circ} \mathrm{C}$ with a Ga-flux $\left(\Phi_{\mathrm{Ga}}\right)$ of $7 \mathrm{~nm} / \mathrm{min}$ and an $\mathrm{N}$-flux $\left(\Phi_{\mathrm{N}}\right)$ of $5 \mathrm{~nm} / \mathrm{min}$. The coalescence of the GaN islands formed in the early stage of the growth can clearly be seen. Figure $1 \mathrm{~b}$ shows the top view image of self-assembled GaN NRs grown at $800^{\circ} \mathrm{C}$ with $\Phi_{\mathrm{Ga}}=4.3 \mathrm{~nm} / \mathrm{min}$ and $\Phi_{\mathrm{N}}=14 \mathrm{~nm} / \mathrm{min}$. It can be seen that only partial coalescence of some NRs takes place. Figure 1c shows the cross-section SEM image of sample in Figure $1 \mathrm{~b}$ where NRs have an average height of $1.2 \mu \mathrm{m}$ and are 


\section{E. Calleja et al.}

$40 \mathrm{~nm}$ in diameter. Low temperature PL spectra of GaN samples shown in Figure 2 reveal strong differences depending on crystal quality. For NRs the spectrum is dominated by the $\mathrm{D}^{0} \mathrm{X}$ line at $3.47 \mathrm{eV}$ and the TES (Two-Electron Satellite) at around $3.45 \mathrm{eV}$. The line width of the $\mathrm{D}^{0} \mathrm{X}$ is around 4-5 meV indicating a high "optical quality" of the NRs. In contrast PL from GaN layer shows a clear peak at $3.45 \mathrm{eV}$ with a line width of $44 \mathrm{meV}$, revealing both the presence of tensile strain and a much lower material quality.

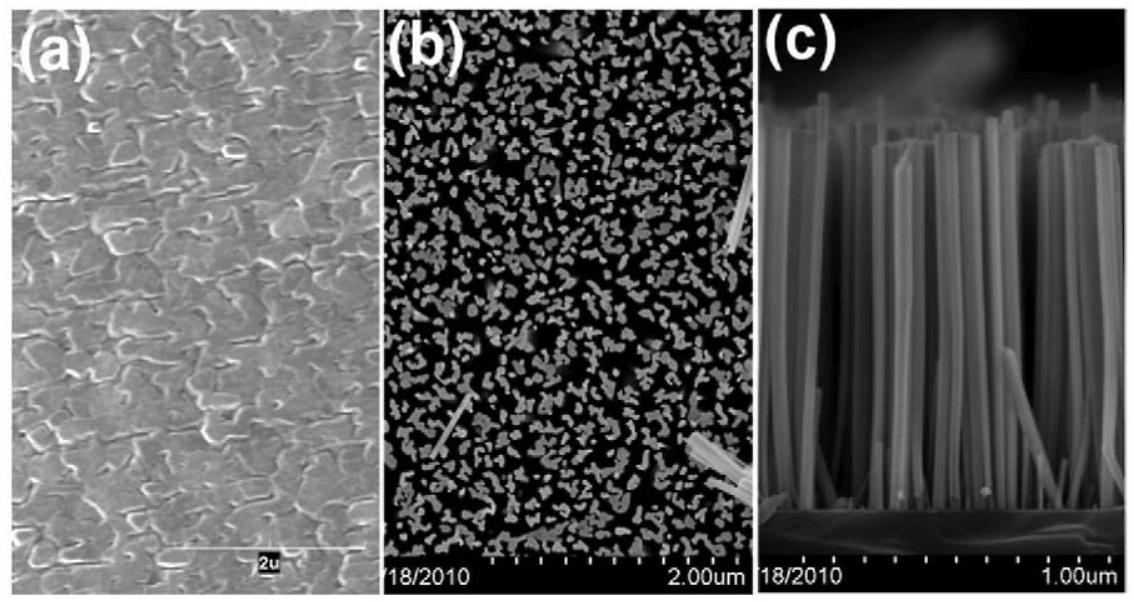

Fig. 1. SEM images of a) a GaN layer (top view), b) GaN NRs (top view), and GaN NRs (cross-section).

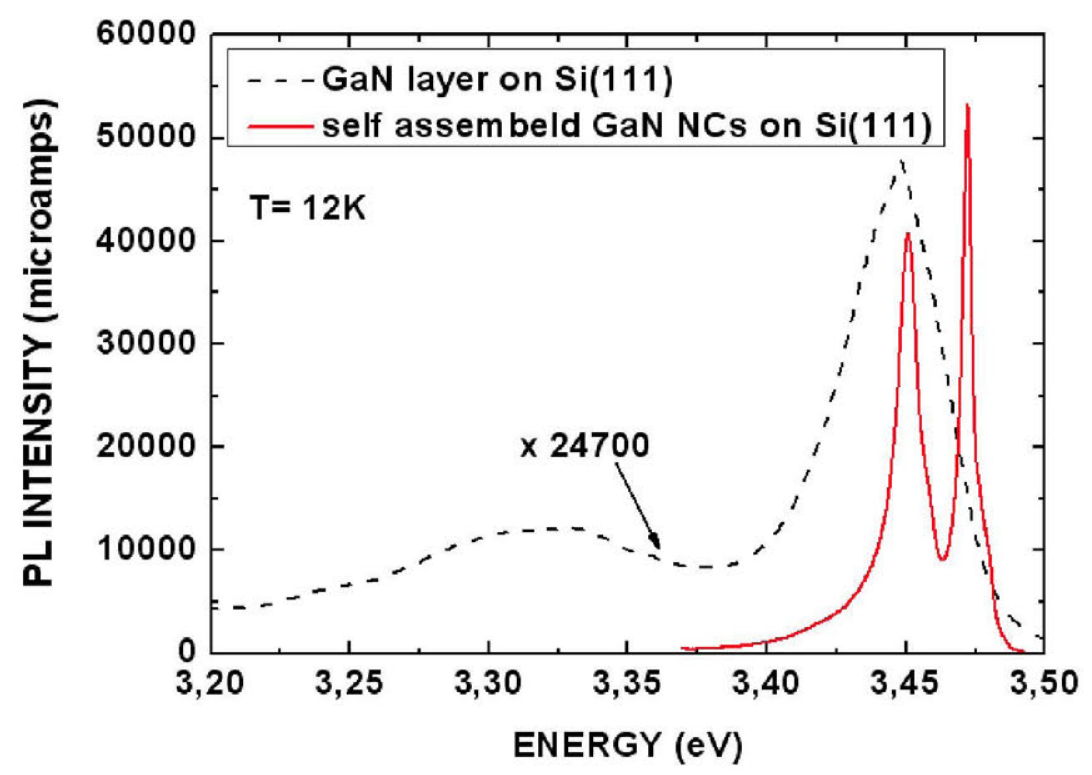

Fig. 2. PL spectra of a GaN layer (dashed line) and GaN NRs (solid line). 


\subsection{Growth of self-assembled InGaN/GaN nanorods on Si(111) substrates}

A successful growth of $\mathrm{InGaN} / \mathrm{GaN}$ nanocolumnar structures needs a previous understanding of the mechanisms that control the incorporation of $\mathrm{In},{ }^{14}$ that mainly depends on: (i) In and Ga fluxes, (ii) active nitrogen amount, and (iii) growth temperature. Points (i) and (ii) reflect the fact that the Ga affinity to bind a nitrogen atom forming GaN is higher than that of In to form InN. Thus, a high In/Ga ratio as well as a high amount of active nitrogen would favor the incorporation of In. Figure 3 shows a clear PL peak shift from $2.56 \mathrm{eV}$ to $2.44 \mathrm{eV}$ when increasing the active nitrogen amount while keeping all other growth parameters fixed.

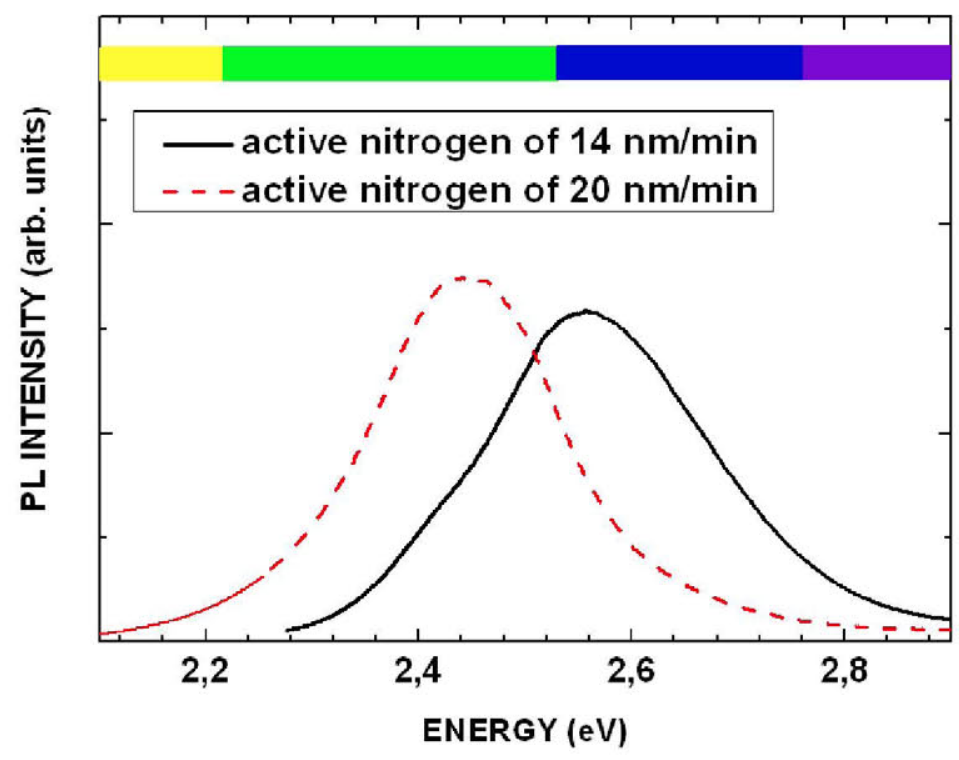

Fig. 3. PL spectra of InGaN NRs grown with different active nitrogen amounts.

Among the three variables mentioned before, the substrate temperature is by far the most important to successfully grow InGaN NRs since it affects the Ga and In desorption and diffusion processes as well as the decomposition of GaN and InN. It is important to note that the desorption of In and the decomposition of $\mathrm{InN}$ are significantly higher than the corresponding to $\mathrm{Ga}$ and $\mathrm{GaN}$ at the typical temperatures to grow self-assembled GaN NRs. ${ }^{25,33}$ Then the approach is to reduce the growth temperature for InGaN NRs to values compatible with the InN decomposition and In desorption rates. In order to study this effect of the growth temperature, a series of InGaN NCs were grown following a two-step method: (i) 1 hour growth of $\mathrm{GaN} \mathrm{NCs}$ at $800^{\circ} \mathrm{C}$, with $\Phi_{\mathrm{Ga}}=4.3 \mathrm{~nm} / \mathrm{min}$ and $\Phi_{\mathrm{N}}=14 \mathrm{~nm} / \mathrm{min}$; and (ii) 1 hour growth of InGaN NCs on top of the GaN NCs, at different fixed temperatures $\left(650^{\circ} \mathrm{C}, 675^{\circ} \mathrm{C}, 700^{\circ} \mathrm{C}, 725^{\circ} \mathrm{C}\right.$ and $\left.750^{\circ} \mathrm{C}\right)$, keeping the same previous $\Phi_{\mathrm{Ga}}, \Phi_{\mathrm{N}}$ values and $\Phi_{\mathrm{In}}=\Phi_{\mathrm{Ga}}$. In order to estimate effects of the InGaN growth 


\section{E. Calleja et al.}

on the NR morphology a reference sample of GaN NRs was grown for two hours under the same conditions as the GaN part of the InGaN/GaN NRs.

Figure 4 shows the In incorporation in these InGaN/GaN NRs as a function of the temperature derived from PL measurements. It can clearly be seen that for lower growth temperatures the In incorporation is increased up to a point of saturation (around $675^{\circ} \mathrm{C}$ ), i.e. all the In supplied is incorporated. The In content can be estimated from the InGaN PL peak energy position according to $\mathrm{Wu}$ et al., ${ }^{34}$ being $\sim 15 \%$ at $725^{\circ} \mathrm{C} ; 22 \%$ at $700^{\circ} \mathrm{C}$ and $33 \%$ at $675^{\circ} \mathrm{C}$ and $650^{\circ} \mathrm{C}$.

As pointed out at the beginning of this section, diffusion and desorption are important variables which are strongly affected by the temperature. As a consequence significant changes in the NRs morphology should be expected as a function of the growth temperature. Indeed, two distinct effects are observed when lowering the growth temperature: (i) a change of growth rate, and (ii) an increase in the NR diameter.

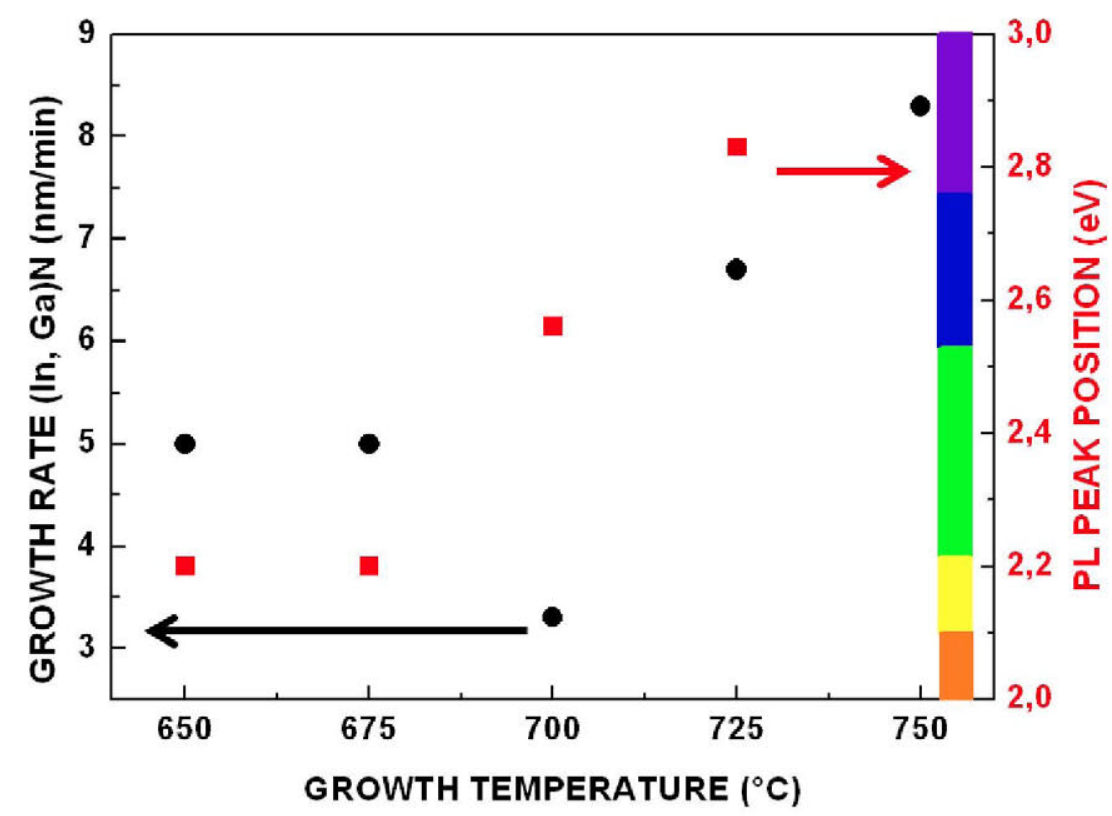

Fig. 4. Low temperature PL peak position and FWHM of InGaN/GaN NRs grown at different temperatures (red squares). Black circles show the growth rate dependence of the InGaN section of NRs on temperature. Reprinted with permission from S. Albert, A. Bengoechea-Encabo, P. Lefebvre, M. A. Sanchez-Garcia, E. Calleja, U. Jahn, A. Trampert, Emission control of InGaN nanocolumns grown by molecular-beam epitaxy on Si(111) substrates, Appl. Phys. Lett. 99, 131108 (2011).

When neglecting the nucleation time, the growth rate of the GaN NRs reference sample can be estimated from Figure 5 a to be around $10 \mathrm{~nm} / \mathrm{min}$. As shown in Figure $5 \mathrm{~b}$, for the InGaN NRs grown at $700^{\circ} \mathrm{C}$ a decrease in total NR length $(1.0$ versus $1.2 \mu \mathrm{m})$ and an increase in diameter ( $100 \mathrm{~nm}$ versus $50 \mathrm{~nm}$ ) as compared to the reference sample can 


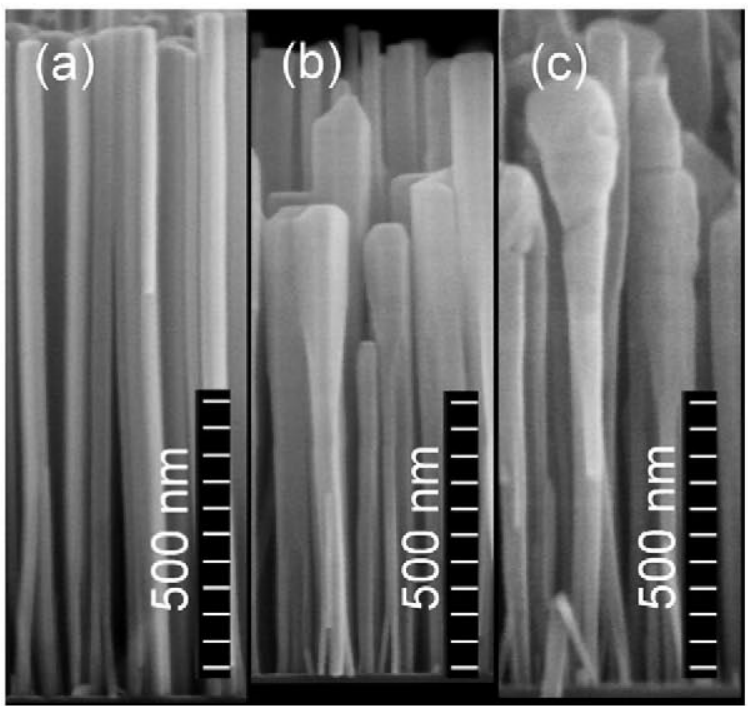

Fig. 5. SEM pictures of: (a) GaN NCs reference sample (growth at $800^{\circ} \mathrm{C}$ ); (b) and (c) are selected InGaN/GaN NCs samples of series A with $\mathrm{InGaN}$ grown at $700^{\circ} \mathrm{C}$ and $650^{\circ} \mathrm{C}$. Reprinted with permission from S. Albert, A. Bengoechea-Encabo, P. Lefebvre, M. A. Sanchez-Garcia, E. Calleja, U. Jahn, A. Trampert, Emission control of InGaN nanocolumns grown by molecular-beam epitaxy on Si(111) substrates, Appl. Phys. Lett. 99, 131108 (2011).

be observed. From cross sectional SEM the growth rate of the InGaN part of the InGaN/GaN NRs can directly estimated for each growth temperature.

These values are plotted in Figure 4, indicating an initial decrease in the InGaN growth rate when lowering the growth temperature from 750 to $700^{\circ} \mathrm{C}$, and a partial recovery for temperatures below $700^{\circ} \mathrm{C}$. The total metal flux $\left(\Phi_{\mathrm{Ga}}+\Phi_{\mathrm{In}}\right)$ being present during the growth of the InGaN NR part can be considered to be close to $\Phi_{\mathrm{Ga}}$ in the temperature range between 750 and $700^{\circ} \mathrm{C}$ due to the very strong In desorption, as indicated by the PL peak position (Figure 4). ${ }^{25}$ With that in mind the reduction of the Gaadatoms diffusion length when lowering the sample temperature may account for the observed reduction in growth rate. Please note that it has been shown previously that the growth rate of GaN NRs is limited by the Ga-flux and that the diffusion of Ga-ad-atoms up the NR sidewalls is the main Ga source for the self-assembled GaN NR growth. ${ }^{24,30}$

Based on these arguments the partial recovery of the growth rate shown in Figure 4 can be explained by an enhanced incorporation of In due to a lower In desorption and InN decomposition.

The increase in the diameter of the NRs is observed to take place below the nominal $\mathrm{GaN} / \mathrm{InGaN}$ interface, with the NR gradually widening up to the top (Figure 4b). From cathodoluminescence date (not shown) it can be seen the emissions related to InGaN arise only from material above this interface pointing towards Ga ad-atoms as the responsible for the diameter increase below the nominal interface. This can be explained 


\section{E. Calleja et al.}

by a longer residence time of Ga ad-atoms on the NR sidewall increasing the probability for them to bind with nitrogen before desorbing or reaching the NR top. Above the nominal GaN/InGaN NR interface both In and Ga will contribute to the lateral growth. When decreasing the growth temperature further the diameter increase can lead to the coalescence of the NRs (Figure 4c).

As discussed before, self-assembled InGaN NRs show in general a polychromatic PL emission mainly due to fluctuations of In \%. This fact, together with inhomogeneities on the current injection (electroluminescence), the appearance of black spots due to defects formation upon NRs merging, and the difficulty to process them in a planar fashion, prevents a commercial exploitation for LEDs, particularly for monochromatic ones. ${ }^{15,16}$ However, self-assembled InGaN NRs are quite helpful to study different strategies to generate white light. Since the growth temperature is one of the main factors that control the In incorporation, a temperature gradient during the InGaN NR growth should lead to a broad emission (white).

Figure 6 shows PL emission from an InGaN/GaN NRs array (self-assembled) where the InGaN region was graded in composition by changing the temperature from $750^{\circ} \mathrm{C}$ to $650^{\circ} \mathrm{C}$, leading to an approximated change in average In $\%$ from 0 to $33 \%$ (Figure 4). PL emission at low temperature is indeed broad (white) and rather intense. However, the PL intensity quenches significantly at room temperature, being this effect much stronger on the "high energy" side of the spectrum.

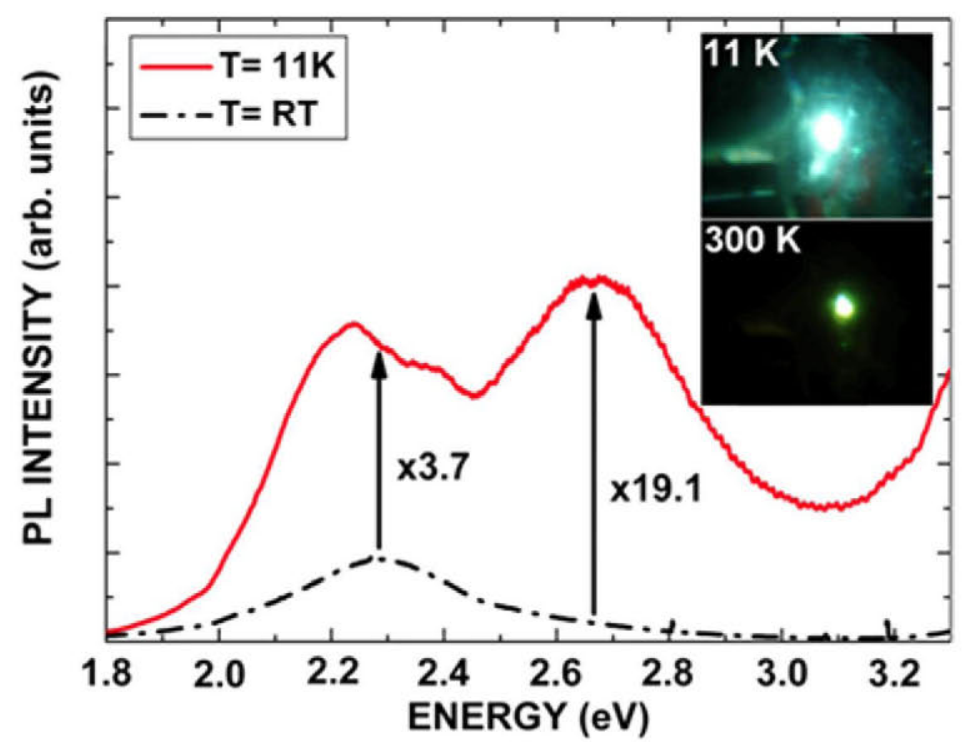

Fig. 6. Room- and low temperature PL of InGaN/GaN NRs grown with a temperature gradient. Reprinted with permission from S. Albert, A. Bengoechea-Encabo, P. Lefebvre, M. A. Sanchez-Garcia, E. Calleja, U. Jahn, A. Trampert, Emission control of InGaN nanocolumns grown by molecular-beam epitaxy on $\mathrm{Si}(111)$ substrates, Appl. Phys. Lett. 99, 131108 (2011). 
This result may be explained by the diffusion of carriers from regions of lower In content (lower localization energy) towards regions of higher In content [17].

\subsection{Growth of InGaN single quantum disks embedded into self-assembled GaN NRs}

Other approach to increase the efficiency of InGaN/GaN self-assembled nanocolumnar heterostructures for light emission is the use of InGaN QDiscs embedded into a GaN NR, leading to an increase in oscillator strength due to a higher confinement. The InGaN QDisc chosen for the confinement study was grown under the same conditions, in terms of absolute III/V ration and $\mathrm{In} / \mathrm{Ga}$ ratio, as the InGaN NRs discussed in the previous section. Having in mind that the InGaN NRs already showed a complete incorporation of supplied In when grown at $675^{\circ} \mathrm{C}$, and assuming a similar behavior when growing QDiscs, these last were grown at $625^{\circ} \mathrm{C}$ while all other conditions were kept constant a rough estimation of the In\% in the QDisc, without considering possible strain related effects, can be derived from PL data, being around $33 \%$, as derived from Figure $4 .^{34}$
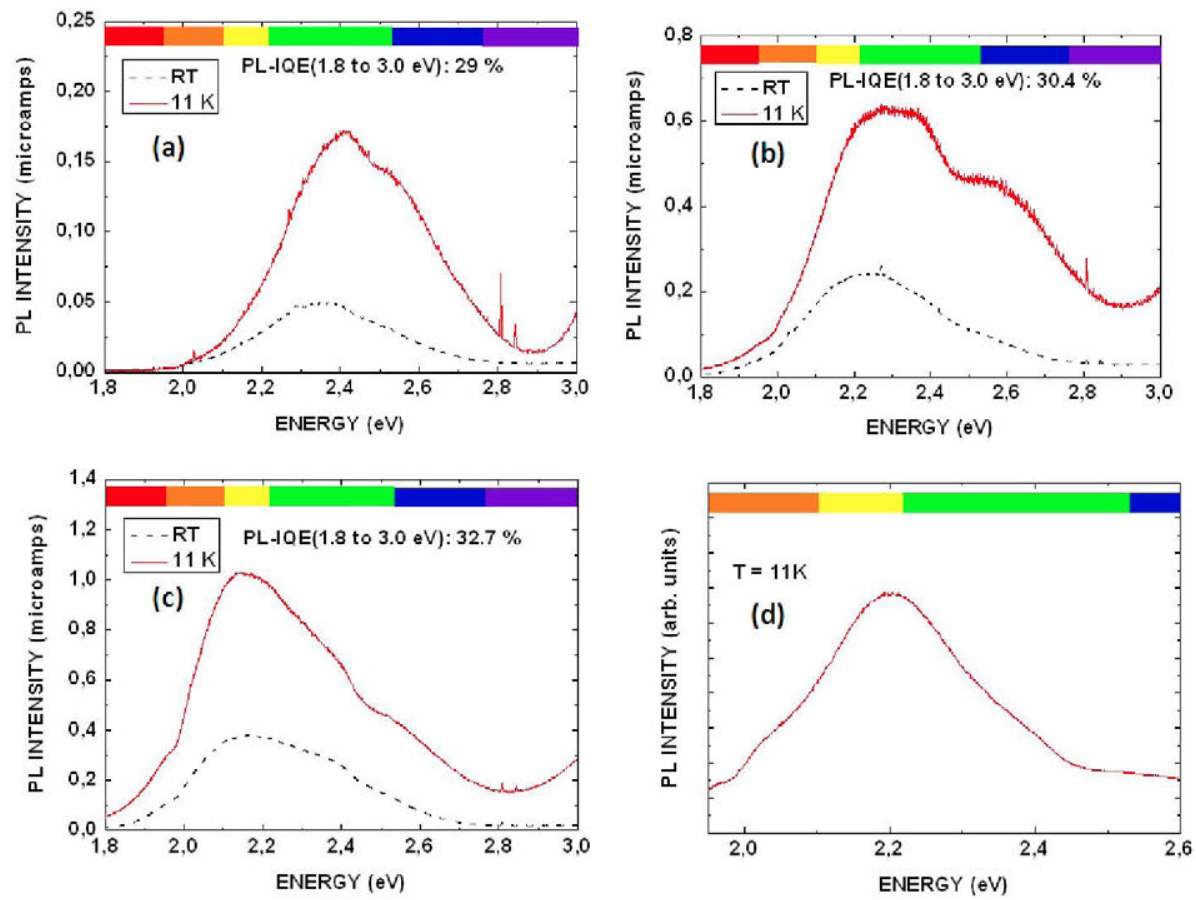

Fig. 7. Room- low temperature PL spectra of single InGaN quantum disk with a nominal thickness of a) $4 \mathrm{~nm}$, b) $8 \mathrm{~nm}$, and c) $16 \mathrm{~nm}$ in comparison to d) low temperature PL spectra of bulk InGaN/GaN NR with InGaN grown at $675^{\circ} \mathrm{C}$.

A series of 3 samples with nominal QDisc thicknesses of $4 \mathrm{~nm}, 8 \mathrm{~nm}$, and $16 \mathrm{~nm}$ was characterized by PL. Figure 6 shows the PL spectra of these samples at RT and $11 \mathrm{~K}$ in comparison to a bulk InGaN/GaN NR sample with the InGaN part grown at $675^{\circ} \mathrm{C}$. For all three QDisk samples an InGaN related PL emission consisting of several peaks can be 
observed. As expected in terms of quantum confinement a blue shift of the overall InGaN related PL emission compared to the InGaN/GaN NR sample (Figure 6d) can be observed for a nominal QDisk thickness of $4 \mathrm{~nm}$ and $8 \mathrm{~nm}$. For the nominally $16 \mathrm{~nm}$ thick QDisk no confinement is visible. For all three samples PL internal quantum efficiencies (PLIQE) of around $30 \%$ are reached.

\subsection{Use of colloidal lithography to generate nanohole masks}

In order to achieve SAG and fabricate ordered arrays of NRs, a nanohole mask must be fabricated on the GaN/sapphire templates, generally obtained by e-beam lithography (EBL). Another technique that provides cheap, fast and almost periodical geometries is the colloidal lithography. In this section a discussion of both masking approaches is presented with special focus on the colloidal lithography. The mask material can be either metal $(\mathrm{Ti}, \mathrm{Mo})$ or dielectric $\left(\mathrm{SiO}_{2}, \mathrm{Si}_{3} \mathrm{~N}_{4}\right)$, but this study will focus on Ti masks

In EBL the mask manufacture consists of a thin Ti film deposition, e-beam patterning of ordered arrays of nanoholes, and transfer of the pattern to the mask underneath by dry etching. To obtain optimal results, additional cleaning steps have to be performed after each of these steps. The most critical aspects that affect the mask surface are roughness, cleanliness, and adhesion of the Ti film to the GaN substrate. When all these parameters are optimized, high quality nanomasks are obtained yielding to a successful and reproducible ordered nanocolumnar growth. These aspects have been thoroughly discussed elsewhere. ${ }^{35}$

Besides EBL, colloidal lithography was used to fabricate Ti masks. As already mentioned, colloidal lithography is an easy, fast and cheap technique compared with others. ${ }^{36,37}$ Though not having a perfect periodicity, these masks are easily fabricated over wide areas ( 2 inch or more) in a short period of time, as compared to EBL, and they allow the study of the SAG process and optimization that will be then transferred to a more standard patterning technique like Nanoimprint Lithography (NIL).

Colloidal lithography is based on the deposition of nanobeads on the GaN surface to be masked by Ti. First the GaN surface is pre-treated by a sequential immersion in solutions of poly-electrolytes negative (PSS) and positively-charged (PDDA). The result is a trilayer PSS/PDDA/PSS, a few nanometers thick, covering the GaN surface. The negatively charged colloidal nanobeads used are made of polystyrene (PS) with diameters around $270 \mathrm{~nm}$.

The solution (4\% in weight) is deposited on the pre-treated GaN surface by spinning. As a result, areas with one monolayer of hexagonal close-packed nanobeads on the surface (scheme in Figure 7a, and example by SEM picture in Figure 7e) are achieved. A following oxygen plasma treatment reduces the diameter of the nanobeads, while keeping the distance between them (pitch) constant (Figure $7 \mathrm{~b}$ ). Then, $7 \mathrm{~nm}$ of Ti are thermally evaporated on the decorated surface (Figure 7c). Finally, nanobeads are removed from the surface, by tape stripping and organics based cleaning, leading to a GaN surface masked by a $7 \mathrm{~nm}$ Ti layer with nanoholes (scheme in Figure $7 \mathrm{~d}$ and SEM picture of a real mask on GaN in Figure 7f). 

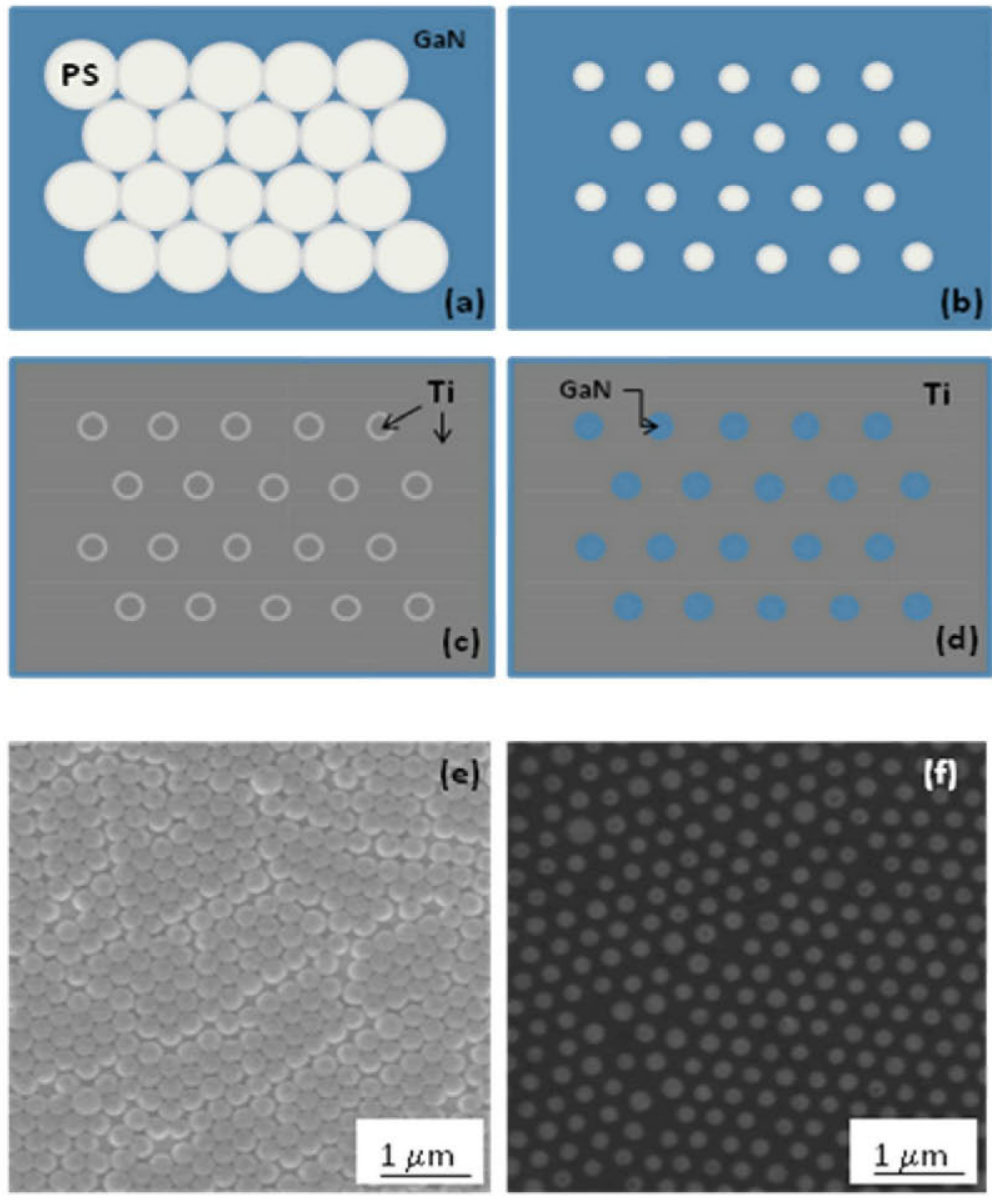

Fig. 8. Steps in colloidal lithography. (a) pre-treated GaN surface is spin coated with PS nanobeads, forming a monolayer with hexagonal symmetry. (b) PS/GaN is treated by oxygen plasma in order to reduce the diameter of nanobeads, and separate them from each other. (c) Ti metallization and (d) removal of the nanobeads (liftoff), leading to the Ti mask on GaN. Figures (e) and (f) show SEM pictures of a hexagonal close-packed PS nanobeads monolayer on $\mathrm{GaN}$, and the resulting Ti mask on $\mathrm{GaN}$, respectively.

\subsection{Growth of ordered NRs on c-plane and a-plane GaN/sapphire substrates}

SAG of GaN NRs needs to achieve simultaneously: (i) selectivity, and (ii) onset of NRs on each nanohole. Since both steps are interconnected and dependent on the selected growth conditions (temperature, III/V) as well as on the mask geometry and material used, it seems plausible that the optimal growth conditions for SAG may differ considerably from those applicable to self-assembled growth. Indeed, these differences are mainly a much higher growth temperature and a very high Ga flux to compensate a huge desorption from the substrate surface. This can be understood based on the requirements to fulfill selectivity. 


\section{E. Calleja et al.}

Selectivity means that no growth must take place on the mask, but only within the nanoholes defined by the mask. In order to reach selectivity, the Ga atoms impinging on the mask must either desorb from it, or diffuse to the nearest nanohole, that is, avoiding nucleating GaN on the mask. This explains the "odd" conditions needed as compared to the self assembled growth. Both a high temperature and low active nitrogen act in the same way when thinking in terms of selectivity.

A high temperature is needed in order to increase both the diffusion length of Ga on the mask and also the Ga desorption rate from it to reduce the probability to nucleate $\mathrm{GaN}$ on it. This means a delicate balance because a too high temperature will certainly avoid nucleation on the mask by the strong Ga desorption, but it may also hinder any growth within the nanoholes. On the other hand, for a given temperature, selectivity will be enhanced for a lower amount of active nitrogen because the probability for Ga atoms to bind a $\mathrm{N}$ atom while on the mask are reduced. Again this means a balance because a low nitrogen amount may considerably reduce the NRs growth rate within the nanoholes.

Finally, the mask geometry is also a factor that must be considered for optimal SAG. The reason being that for a given temperature and nitrogen amount the distance between nanoholes (pitch), if too long, may favor GaN nucleation on the mask. This will happen if the pitch is too long in respect to the Ga atoms diffusion length (that depends on temperature). In addition, the size of nanoholes (diameter) for a given pitch will change the total density of $\mathrm{Ga}$ atoms within them, because of the $\mathrm{Ga}$ arriving by diffusion along the mask.
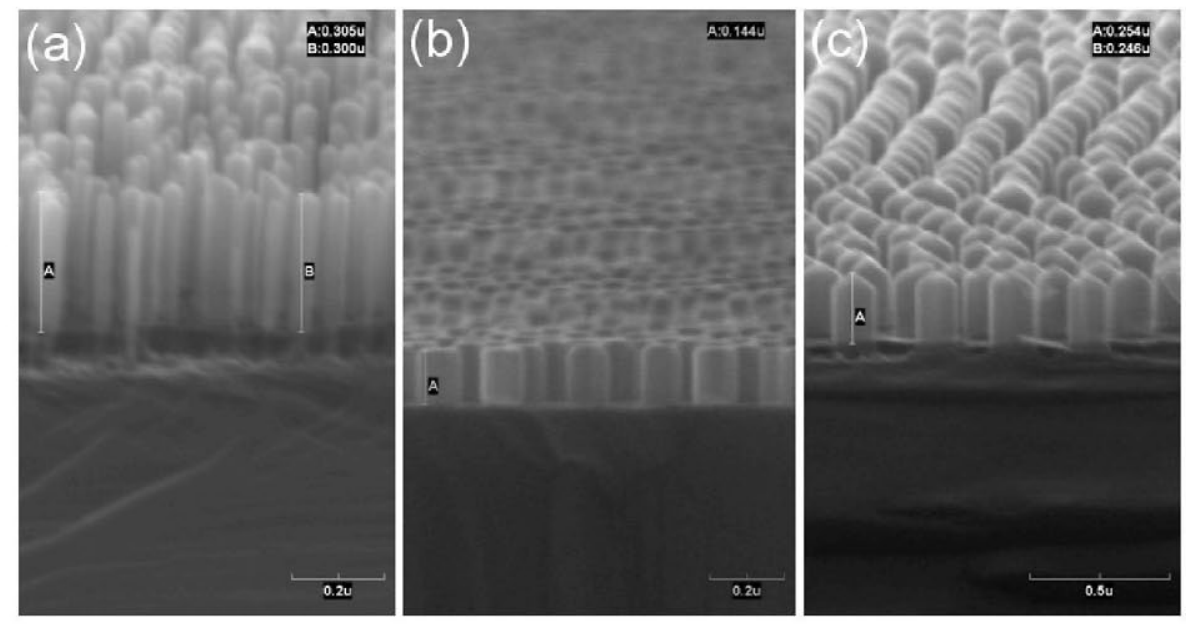

Fig. 9. SEM images of orderd GaN NRs with different diameters: a) $50 \mathrm{~nm}$, b) $100 \mathrm{~nm}$, and c) $150 \mathrm{~nm}$.

Having all these factors in mind, ordered NRs of diameters from few tens of nanometers to almost one micron can be grown selectively. ${ }^{19,20,21,22}$ In this work, ordered arrays of GaN NRs with diameters ranging from $50 \mathrm{~nm}$ to $150 \mathrm{~nm}$ were successfully grown, as shown in Figure 9, using colloidal lithography. Note that different morphologies of the NR top surface can be distinguished. Namely, pyramidal "pencil 
like" (Figure 9c) or flat (Figure 9b) can be achieved depending on the growth conditions. A detailed discussion of this aspect is going to follow in an upcoming work, though it can be said that the main factor that determines the NR top profile is the density of Ga atoms within the nanohole at the early nucleation stage.

Figure 10 shows SEM images (side and top) of an array of GaN NRs grown, in this case, on a EBL Ti mask. The dispersions in height and diameter with relative standard deviation of $1.6 \%$ and $2.9 \%$ respectively are quite small compared to those observed in self-assembled GaN NRs with standard deviations of $25 \%-60 \%$ in height and $10 \%-45 \%$ in diameter. ${ }^{24,38,39}$
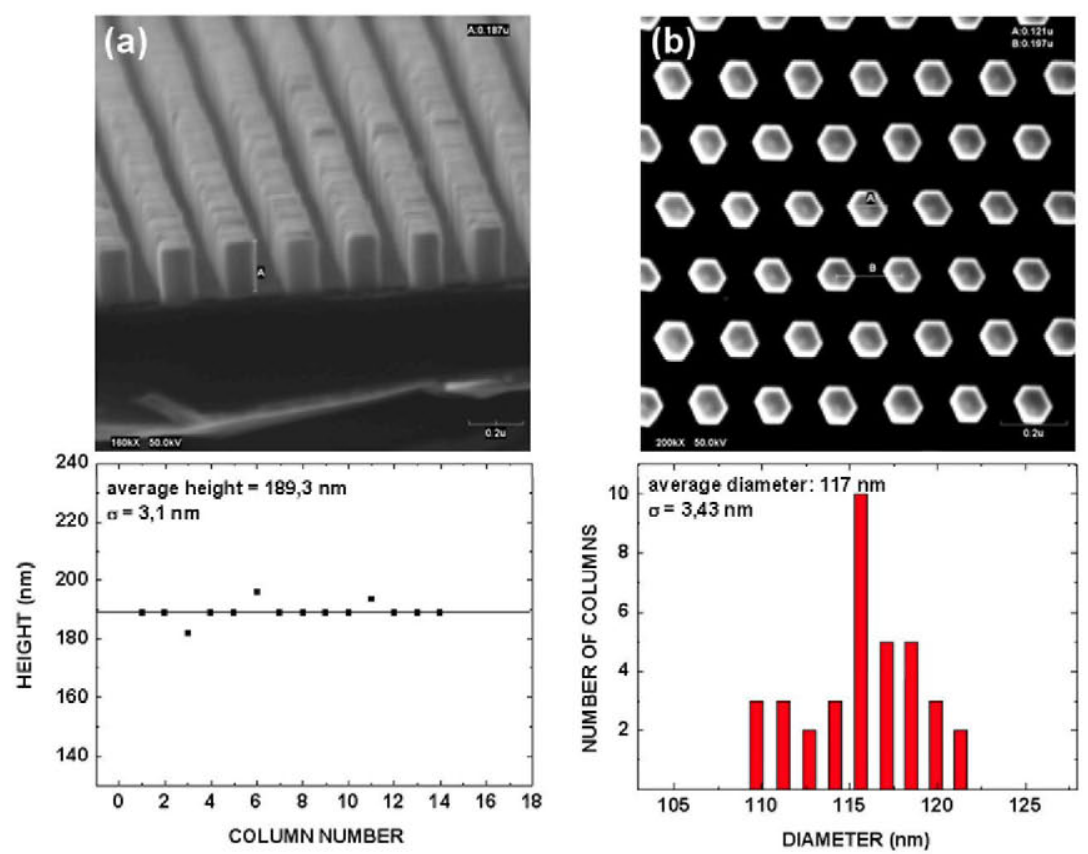

Fig. 10. SEM images of an array of ordered GaN NRs and the corresponding height and diameter distributions: a) side view and height distribution, b) top view and diameter distribution. This array was grown on a EBL Ti mask.

The cross-sectional scanning TEM bright-field image in Figure 10a shows that the diameter of the NR agrees well with the nanohole size defined on the Ti mask. The dark contrast lines at the interface between $\mathrm{NR}$ and $\mathrm{GaN}$ template indicate structural imperfections due to basal plane stacking faults (SF). The presence of stacking faults in the NRs is confirmed by the diffraction contrast image shown in Figure 10b. Besides these SFs no other extended defects, like threading dislocations, are observed. This is proven by the lattice images in Figures $10 \mathrm{c}$ and $10 \mathrm{~d}$ showing that most of the stacking faults cross the whole NR and therefore do not lead to the formation of partial dislocations. Since in SAG of GaN NRs no epitaxial strain is involved (homoepitaxy on GaN templates) these stacking faults could be attributed to impurities such as titanium. 


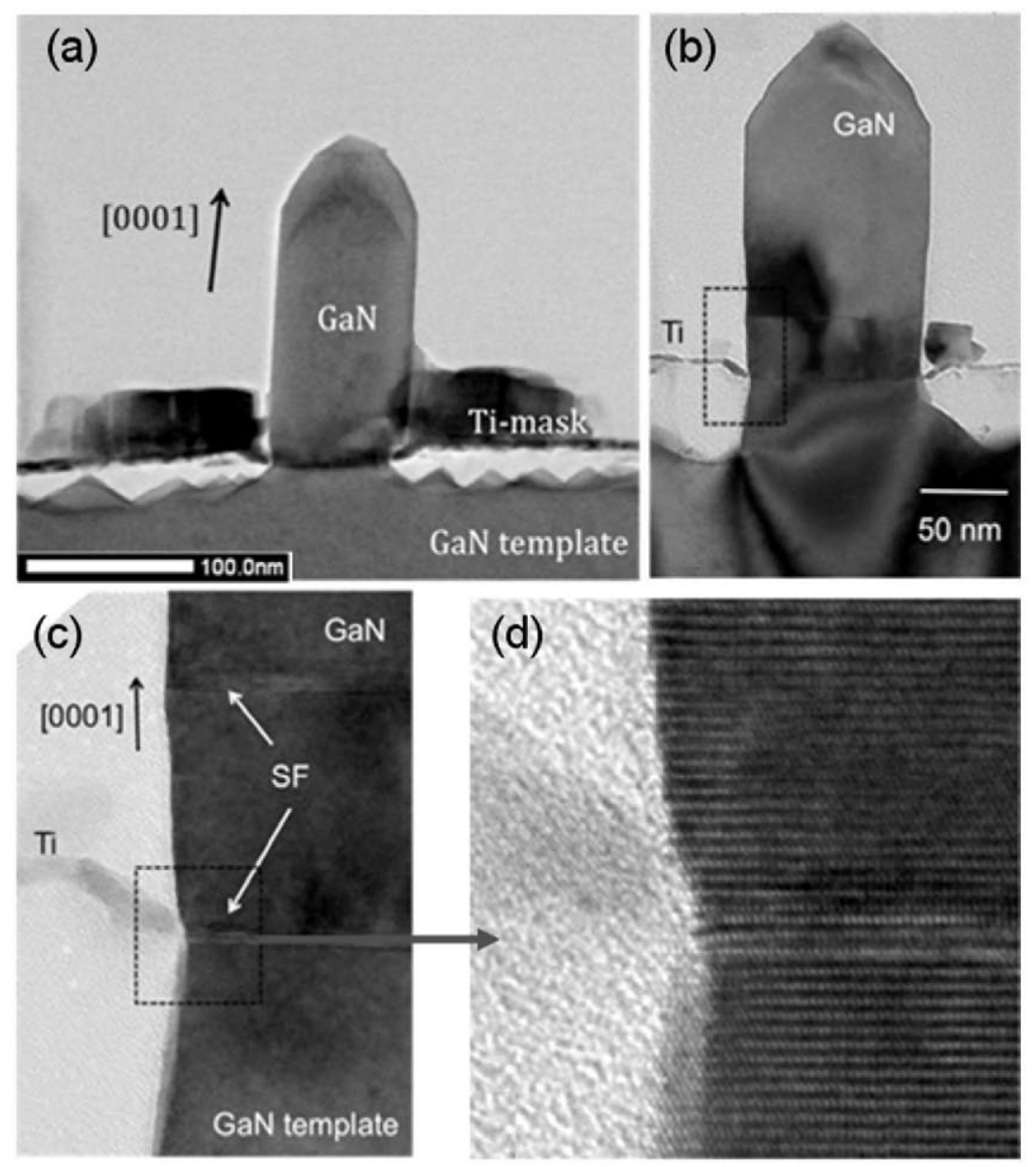

Fig. 11. a) cross-sectional scanning TEM bright-field image, b) cross-sectional scanning TEM diffraction contrast image, and c, d) cross-sectional scanning TEM lattice images of an ordered GaN NR.

In order to evaluate the optical quality of the ordered NRs, PL spectra have been taken at low temperature and compared to those from self assembled NRs, as shown in Figure 11.

Both the self assembled and the ordered NRs show a peak at around $3.47 \mathrm{eV}$ which can be attributed to the $\mathrm{D}^{0} \mathrm{X}$ indicating that in both cases the NRs, as expected, are virtually strain free. ${ }^{4,32}$ The line widths of the $\mathrm{D}^{0} \mathrm{X}$ line for both ordered and self assembled NRs are around $5 \mathrm{meV}$.

Another advantage of SAG to be exploited for broad light emission relies on the control to terminate GaN NRs, either with flat tops, or pyramidal ones. The growth of InGaN QDiscs in such NRs will follow the overall growth front geometry on the GaN NCs, which will lead to QDisc shapes varying from cylindrical shapes to more 


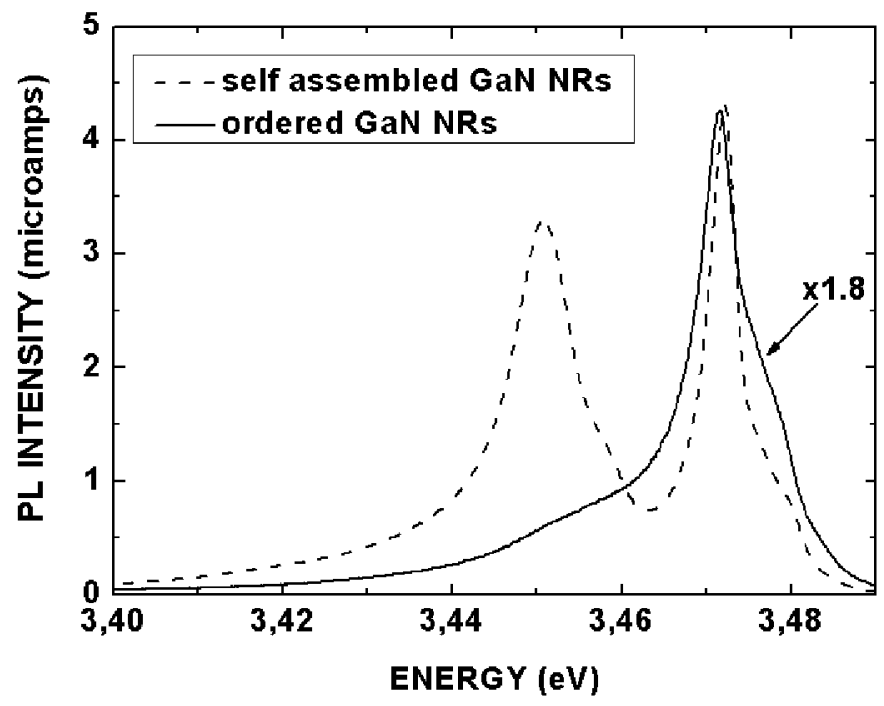

Fig. 12. Normalized PL spectra of ordered NRs (solid line) and self assembled NCs (dashed line) at 11K.

complicated ones involving different planes (polar, non-polar and semi-polar). Since the growth on non-polar or semi-polar planes may reduce or suppress the internal electric field, the problems caused by quantum confined Stark effect may also be reduced or entirely suppressed. ${ }^{40}$

In the case of SAG of GaN NRs on c-plane GaN templates, the NR growth front, though controllable as mentioned before, is in most cases formed by semi-polar planes ( $\mathrm{r}$ planes) and c-plane on top that yields a "pencil-like" profile. 19,20,21,22 When growing an InGaN QDisc on them, a similar or even more complicated profile is generated for the QDisc. $^{21}$

As mentioned before, this kind of heterostructure may improve the light emission broadening (white) as well as its intensity due to a stronger electron-hole wave-functions overlapping. However, another, possibly better, approach is to grow the InGaN QDiscs and GaN NRs directly on non-polar GaN [10-10] (m-planes) or [11-20] (a-planes) planes.

The growth of ordered GaN NRs on $a$-plane GaN/sapphire templates by PAMBE has recently been demonstrated. ${ }^{37}$ The same conditions and critical steps are applicable for the SAG of GaN NRs either on $c$-planes or $a$-planes. Perfect selectivity on colloidal lithography masks is achieved on $a$-plane GaN/sapphire templates, as shown in Figure 12. However, in this case, significant differences concerning the lateral and vertical growth rates can be seen.

For SAG of GaN NRs on a $c$-plane GaN template, hexagonal prisms ending with a pyramidal or flat top are obtained. In the case of SAG on a-plane GaN templates, elongated (lateral) nanostructures with different facets are obtained, being the top most one an $m$-plane, as shown in Figure 13. The direction along which these nanostructures show a pyramidal lateral termination (Figure 12a) corresponds to the $c$-plane. The cross section on Figure 13 was cut along this direction that shows nanostructure elongation. 


\section{E. Calleja et al.}

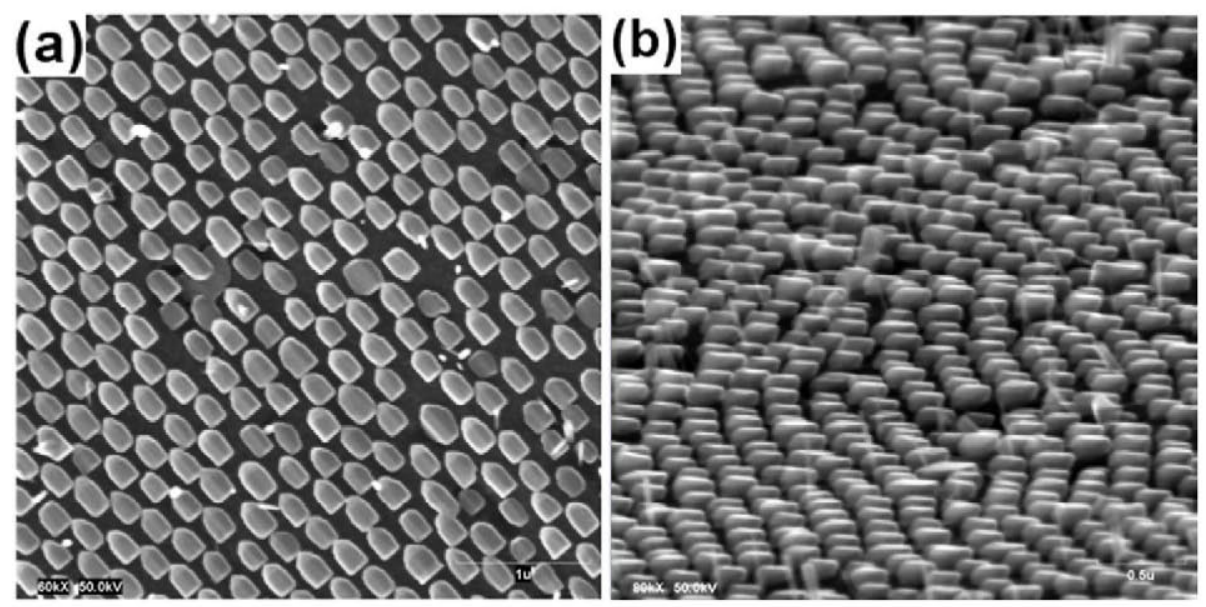

Fig. 13. SEM image of ordered GaN NRs on $a$-plane GaN template: a) top view. b) bird's eye view. a) Reprinted with permission from A. Bengoechea-Encabo, S. Albert, M.A. Sanchez-Garcia, L.L. López, S. Estradé, J.M. Rebled, F. Peiró, G. Nataf, P. de Mierry, J. Zuniga-Perez, E. Calleja, Selective area growth of $a$ and $c$-plane GaN nanocolumns by molecular beam epitaxy using colloidal nanolithography, J. Crystal Growth 353, 1, 1-4 (2012).

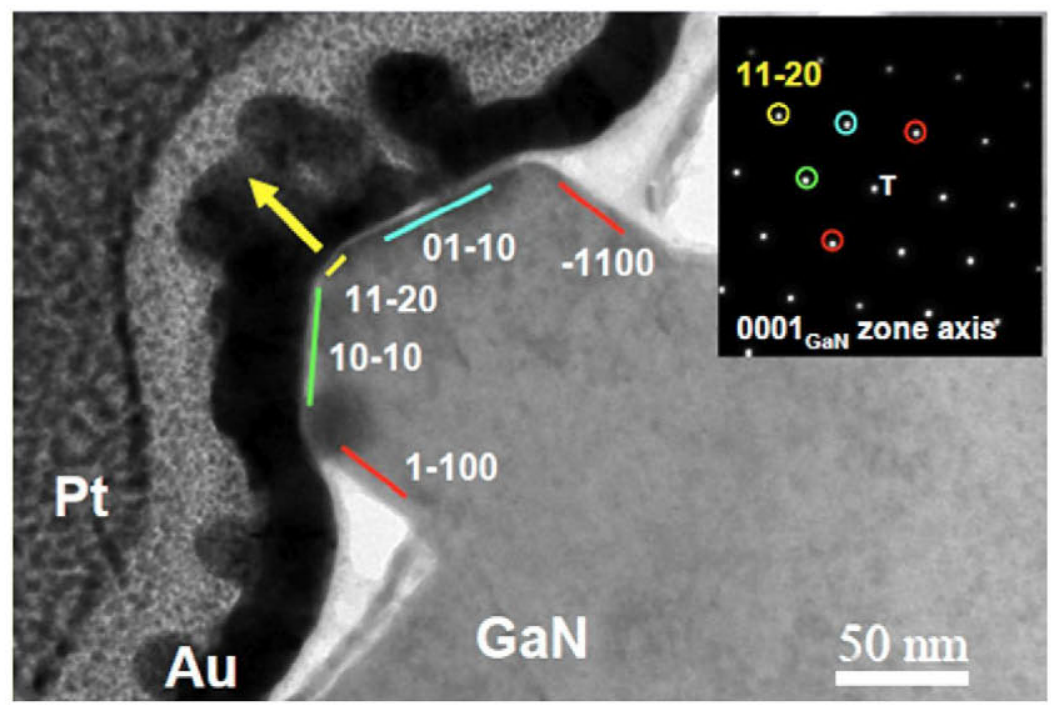

Fig. 14. View of the NR exhibiting facets along planes perpendicular to corresponding g vectors for most of the spots in 0001 axis diffraction pattern (inset). Reprinted with permission from A. Bengoechea-Encabo, S. Albert, M.A. Sanchez-Garcia, L.L. López, S. Estradé, J.M. Rebled, F. Peiró, G. Nataf, P. de Mierry, J. Zuniga-Perez, E. Calleja, Selective area growth of $a$ - and $c$-plane GaN nanocolumns by molecular beam epitaxy using colloidal nanolithography, J. Crystal Growth 353, 1, 1-4 (2012). 
The vertical growth direction is confirmed to be [11-20], perpendicular to the $a$-plane. The nanostructures exhibits lateral faceting perpendicular to [1-100] and top facets perpendicular to [10-10] and [01-10], i.e. m-planes. The corresponding selected area diffraction pattern along the [0001] axis is shown in the inset of Figure 13, indicating that the nanostructure elongation occurs in that direction. Same results have been observed for similar structures grown by MOCVD. ${ }^{41}$

Figure 14 shows the measured vertical and lateral growth rates for GaN NRs grown on $c$-planes and $a$-planes. The growth on c-planes is quite anisotropic, that is, the axial growth rate dominates by far, what leads to well developed NRs with a high aspect ratio. On the other hand, while the NRs lateral growth on $c$-plane is almost zero, it reaches a considerable value when growing on $a$-planes (ratio of 0.4 ). This ratio is kept almost constant when the active nitrogen is increased.

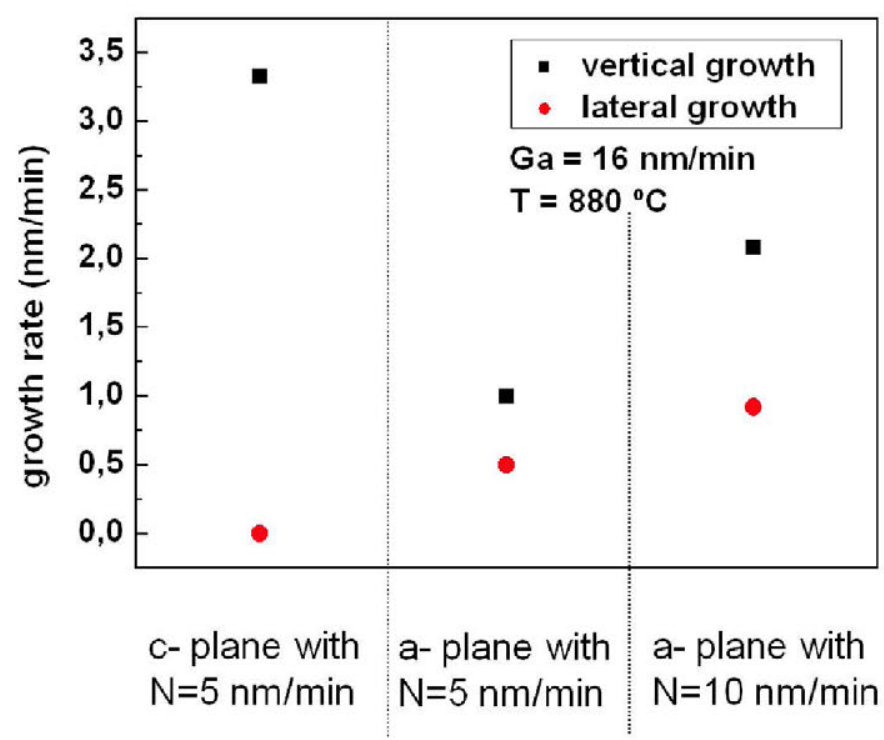

Fig. 15. Vertical and lateral growth rates for GaN NRs SAG on $c$-plane (growth temperature $880^{\circ} \mathrm{C}, \Phi_{\mathrm{Ga}}=16$ $\mathrm{nm} / \mathrm{min}, \Phi_{\mathrm{N}}=5 \mathrm{~nm} / \mathrm{min}$ ), $a$-plane under the same growth conditions, and $a$-plane under twice as much active nitrogen amount $(10 \mathrm{~nm} / \mathrm{min})$. Reprinted with permission from A. Bengoechea-Encabo, S. Albert, M.A. Sanchez-Garcia, L.L. López, S. Estradé, J.M. Rebled, F. Peiró, G. Nataf, P. de Mierry, J. Zuniga-Perez, E. Calleja, Selective area growth of $a$ - and $c$-plane GaN nanocolumns by molecular beam epitaxy using colloidal nanolithography, J. Crystal Growth 353, 1, 1-4 (2012).

In order to evaluate the quality of non-polar SAG GaN NRs, PL spectra have been taken on them, as well as on the $a$-plane GaN template (prior to growth). Figure $15 \mathrm{a}$ clearly shows a strong yellow band and a rather weak emission at around $3.4 \mathrm{eV}$ for the GaN template, indicating a rather low quality. On the other hand, the GaN NRs show no yellow band and a strong band edge emission with two peaks at around 3.36 and $3.42 \mathrm{eV}$. In Figure 15b PL spectra of SAG NRs on $a$-plane and $c$-plane are compared. On $c$-plane, GaN NRs are fully relaxed, and the dominant excitonic emission corresponds to the $\mathrm{D}^{0} \mathrm{X}$ 


\section{E. Calleja et al.}

line (Figure 11). However, on a-plane the dominant line is around $3.42 \mathrm{eV}$, that is redshifted $50 \mathrm{meV}$ in respect to the relaxed $\mathrm{D}^{0} \mathrm{X}$ line. This may indicate a strong biaxial tensile strain for GaN NRs grown on $a$-plane. Actually, the band edge PL line from the GaN template (3.43 eV in Figure 15a) also corresponds to a red-shifted emission most likely due to biaxial tensile strain. It seems that there is a correlation between the two strain states in the template and the grown NRs, something that has never been observed when GaN NRs are grown on $c$-planes. The fact that the GaN NRs grown on $a$-plane are elongated and have a higher footprint as compared to their counterparts grown on cplanes, as well as a much smaller aspect ratio, maybe determinant to keep them under the biaxial tensile strain "inherited" from the template. In other words, a much higher nanostructure may eventually relax. A careful look at the NRs interface with the template by High Resolution TEM, checking for dislocation networks, may shine some light to better interpret these results.
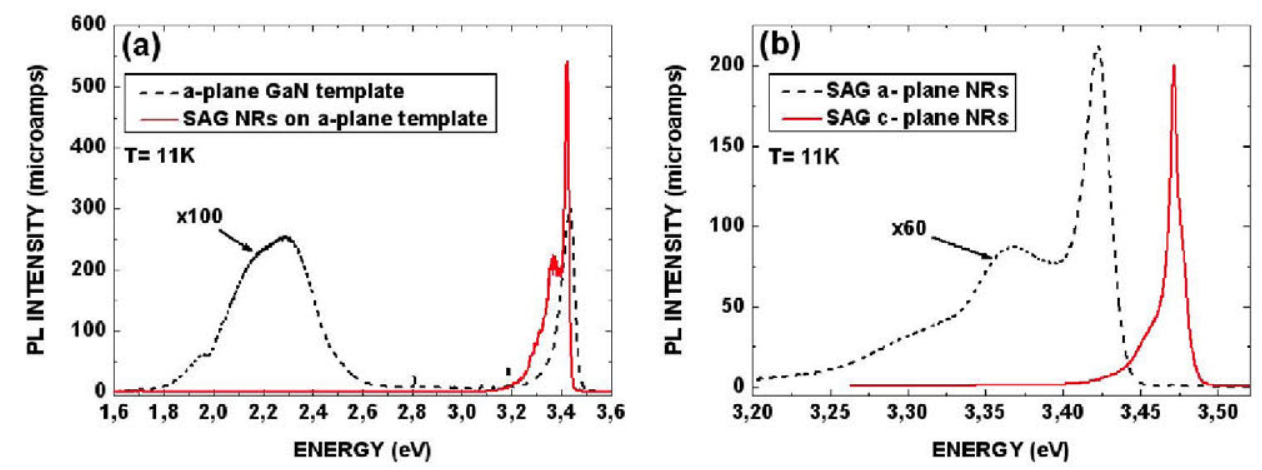

Fig. 16. PL spectra of a) $a$-plane GaN template, b) SAG GaN NRs on the $a$-plane GaN template.

\subsection{Growth of InGaN single quantum disks embedded into ordered GaN NRs}

As in the case of self-assembled NRs, embedded InGaN QDiscs can be grown embedded into ordered NRs, either on $c$-plane, or a-plane. Figure 16 shows the morphology and optical properties of these structures.

The PL spectrum of the InGaN QDiscs grown on $a$-plane SAG NRs shows a dominant emission line at $2.34 \mathrm{eV}$, attributed to the QDisc. The broad shoulder in the range of $2.6 \mathrm{eV}$ to $3.3 \mathrm{eV}$ is most probably related to the growth of InGaN on the TiN mask, as can be seen in Figure 16a (grey contrast between the NRs). The SEM image in Figure $16 \mathrm{~b}$ reveal that the structures grown on $c$-plane GaN/sapphire templateshave a pyramidal top. From the PL spectrume two InGaN related peaks at around $2.45 \mathrm{eV}$ and $2.95 \mathrm{eV}$ are observed. The existence of two emission lines in the PL spectrum of InGaN QDiscs embedded on NRs with pyramidal top has already been reported elsewhere. ${ }^{21}$ The low energy peak is thought to originate from the semi-polar side facet of the pyramidal top, while the high energy peak relates most likely to a polar plane developing at the apex formed by the convergence of six non-polar facets. 


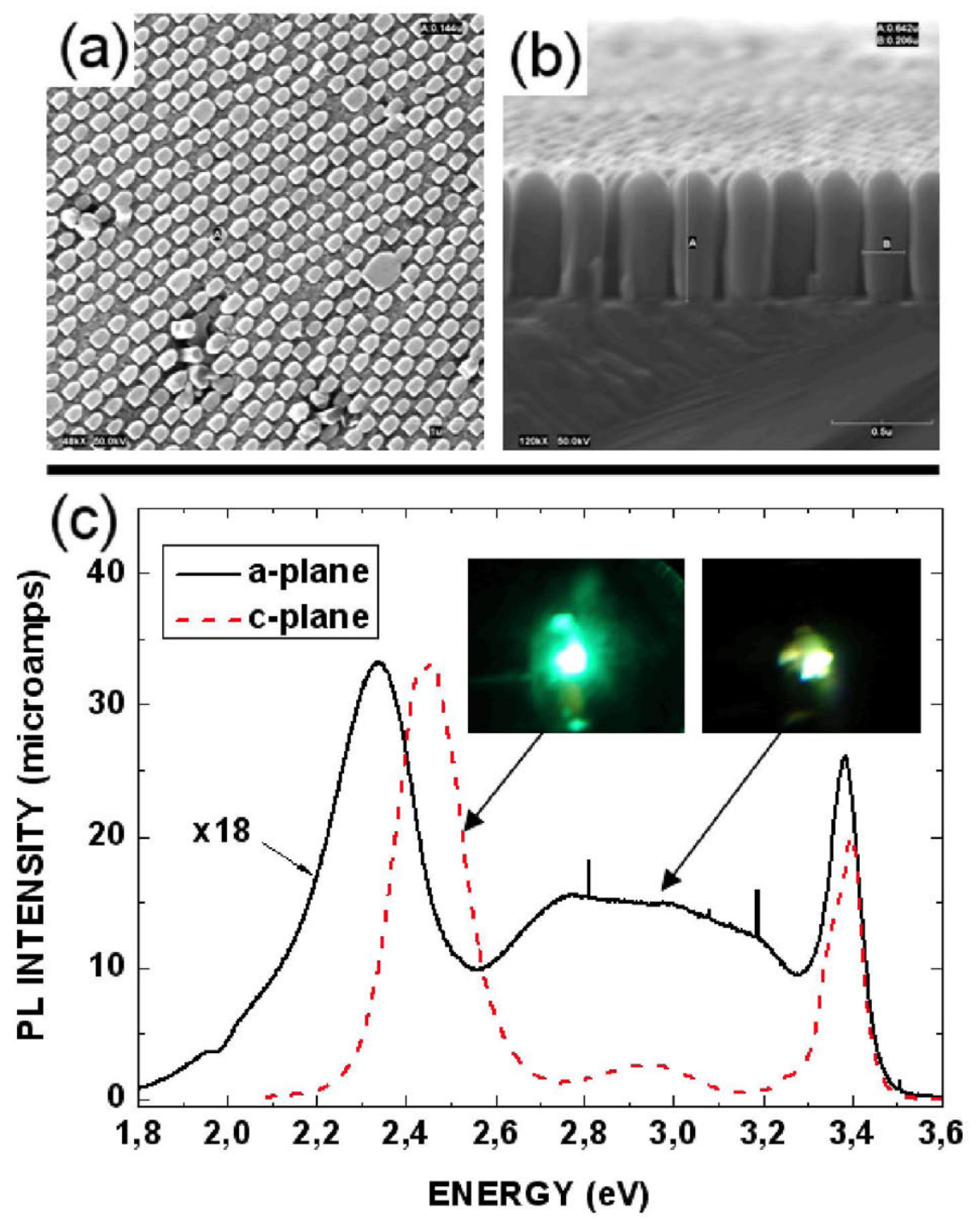

Fig. 17. Comparison of InGaN QDiscs grown on ordered $c$ - and $a$-plane GaN NRs: a) top view SEM image of InGaN/GaN NRs grown on $a$-plane. b) side view SEM image of InGaN/GaN NRs grown on $c$-plane. c) Room temperature PL spectra of $a$ - and $c$-plane InGaN/GaN NRs.

Very high IQE was observed in the case of PL emission from the InGaN nano-disk embedded on a-plane GaN nanostructures. Figure 18 shows the LT $(12 \mathrm{~K})$ and RT PL spectra comparison. The IQE of the overall spectra is 7\%, but as it was explained before, the emission has different InGaN contributions. Although the emission coming from the InGaN grown on the Ti mask (range between $2.3 \mathrm{eV}$ to $3.3 \mathrm{eV}$ ) has a relatively low IQE 


\section{E. Calleja et al.}

(4.7\%), as expected, the one coming from the embedded InGaN on the a-plane nanostructures, corresponding approximately to the range from $1.6 \mathrm{eV}$ to $2.3 \mathrm{eV}$ (red to green), has a IQE as high as $58 \%$. Further improvement in the selective growth on aplane templates promises an extraordinary effect on the performance of future devices.

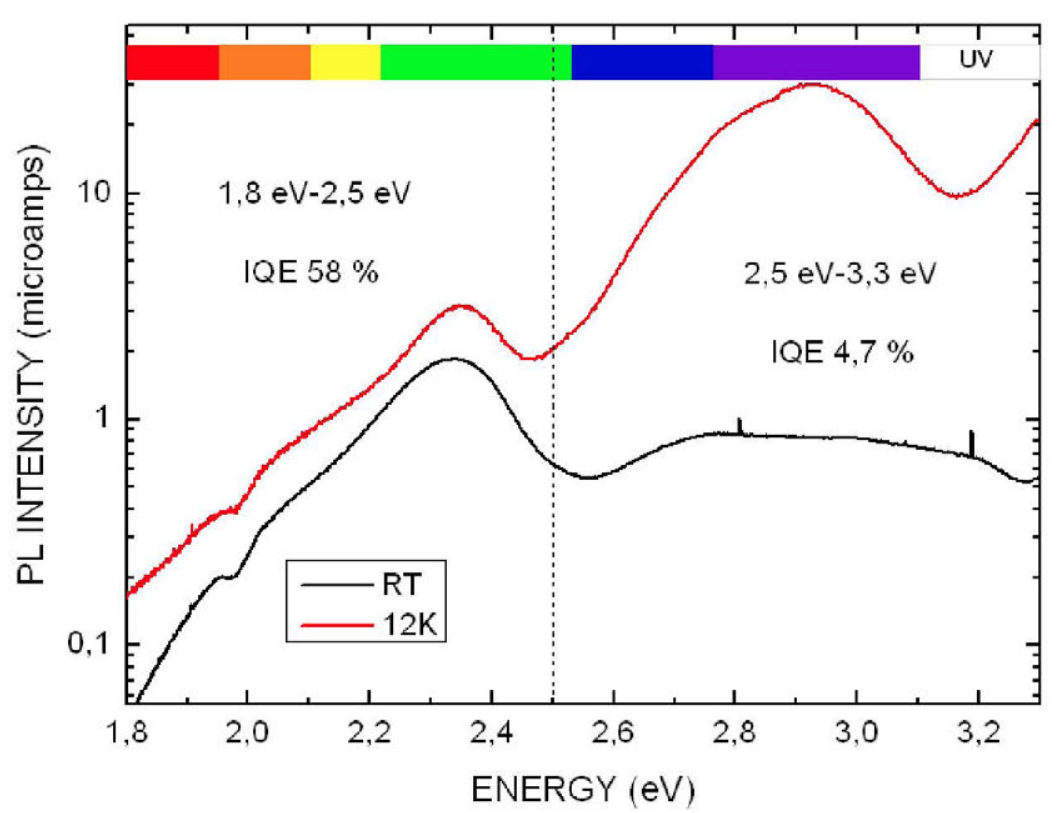

Fig. 18. LT and RT PL spectra of the InGaN nanodisk embedded on a-plane GaN nanostructures. Two different regions are distinguished. The emission at lower energy corresponds to the embedded InGaN nanodisk, with a very high IQE, up to $58 \%$. The emission at higher energies, with lower IQE around $4.7 \%$, corresponds to the InGaN non-selectively grown on the mask.

\section{Summary}

The first part of this work described basic principles involved in the growth of GaN selfassembled NRs grown on $\mathrm{Si}(111)$ substrates by PAMBE. Depending on the III/V ratio and sample temperature either GaN layers or GaN self-assembled GaN nanocolumns grow directly on $\mathrm{Si}(111)$. A significant improvement in optical quality of the selfassembled GaN NRs compared to GaN layers was observed.

Following that, the effect of the growth temperature on the morphology and emission characteristics of self-assembled InGaN NRs was studied. Within the growth temperature range of $650^{\circ} \mathrm{C}$ to $750^{\circ} \mathrm{C}$, an increase in the In incorporation for decreasing temperature was observed. This effect allowed tailoring the InGaN NRs emission line shape by using temperature gradients during growth yielding white emission at room temperature and low temperature. Besides that the implementation of single InGaN QDisk structures into self-assembled GaN NRs was shown, revealing a clear blue-shift with decreasing nominal thickness of the quantum disk. 
The second part of this work described the influence of the substrate temperature, and III/V flux ratio on the SAG of GaN NRs on both c-plane sapphire/GaN- and a-plane sapphire/GaN substrates. Two ways of lithography were presented putting special emphasis on the colloidal lithography, as a method which is able to handle big areas in a short time. With optimized conditions, ordered GaN NRs were grown with a wide variety of diameters. SAG of a-plane GaN NRs was compared, in terms of lateral, vertical growth rates, and optical properties with the SAG of c-plane GaN NRs. The vertical growth rate of ordered a-plane and c-plane nanocolumns was found to differ by a factor of 3.5 , with the same conditions being used. Besides that it was shown that ordered a-plane NRs show a significant lateral growth rate, which is in contrast to the ordered c-plane NRs. For the optical properties it has been found that ordered c-plane NRs are fully relaxed and show a full width half maximum comparable to self-assembled GaN NRs. In the case of ordered a-plane NRs only partial relaxation with respect to the strained a-plane substrate and a higher full width half maximum compared to ordered c-plane GaN nanocolumns was observed. In addition to that the implementation of single InGaN QDisk structures into both ordered a-plane GaN and c-plane GaN NRs was shown revealing green emission for the single quantum disk implemented on ordered c-plane GaN NRs and white emission for the single QDisk implemented in ordered a-plane NRs at room temperature.

Based on the presented results, two lines are being under development in our group. First, following the RGB approach initiated with self-assembled NRs, the ordered growth of c-oriented InGaN NRs achieving red, green and blue emission, and their combination for white light emission. And second, the improvement of selectivity during the InGaN growth on a-plane nanostructures, since first results have shown a very high IQE $(58 \%)$ for the InGaN embedded in such nanostructures.

\section{Acknowledgments}

We acknowledge partial financial support by the EU FP7 Contract SMASH 228999-2; the Initial training network RAINBOW project PITN-GA-2008-213238 and by Spanish projects CAM/P2009/ESP-1503 and MICINN MAT2008-04815.

\section{References}

1. S. Nakamura, G. Fasol, S.J. Pearton, The Blue Laser Diode: The Complete Story (Springer, Berlin, 2000).

2. F.K. Yam, Z. Hassan, InGaN: An overview of the growth kinetics, physical properties and emission mechanisms, Superlattices \& Microstructures 43, 1 (2008).

3. T. Kuykendall, P. Ulrich, S. Aloni, P. Yang, Complete composition tunability of InGaN nanowires using a combinatorial approach, Nat. Mater. 6, 952 (2007).

4. E. Calleja, M.A. Sachnchez-Garcia, F.J. Sanchez, F. Calle, F.B. Naranjo, E. Munoz, U. Jahn, $\mathrm{K}$. Ploog, Luminescence properties and defects in GaN nanocolumns grown by molecular beam epitaxy, Phys. Rev. B 62, 16826 (2000). 
5. R. Meijers, T. Richter, R. Calarco, T. Stoica, H.P. Bochem, M. Marso, H. Lüth, GaN-nanowhiskers: MBE-growth conditions and optical properties, J. Crystal Growth 289, $381(2006)$.

6. N. Thillosen, K. Sebald, H. Hardtdegen, R. Meijers, R. Calarco, S. Montanari, N. Kaluza, J. Gutowski, H. Luth, The state of strain in single GaN nanocolumns as derived from microphotoluminescence measurements, Nano Letters 6, 704 (2006).

7. M. Yoshizawa, A. Kikuchi, M. Mori, N. Fujita, and K. Kishino, Growth of Self-Organized $\mathrm{GaN}$ Nanostructures on $\mathrm{Al}_{2} \mathrm{O}_{3}$ (0001) by RF-Radical Source Molecular Beam Epitaxy, Jpn. J. Appl. Phys. 36, L459 (1997).

8. Y.S. Park, C.M. Park, D.J. Fu, T.W. Kang, J.E. Oh, Photoluminescence studies of GaN nanorods on $\mathrm{Si}$ (111) substrates grown by molecular-beam epitaxy, Appl. Phys. Lett. 85, 5718 (2004).

9. H.-M. Kim, Y.-H. Cho, H. Lee, S.I. Kim, S.R. Ryu, D.Y. Kim, T.W. Kang, K.S. Chung, High Brightness light emitting diodes using dislocation-free indium gallium nitride/gallium nitride multiquantum-well nanorod arrays, Nano Letters 4, 1059 (2004).

10. C.H. Chiu, T.C. Lu, H.W. Huang, C.F. Lai, C.C. Kao, J.T. Chu, C.C. Yu, H.C. Kuo, S.C. Wang, C.F. Lin, T.H. Hsieh, Fabrication of InGaN/GaN nanorod light-emitting diodes with self-assembled Ni metal islands, Nanotechnology 18, 445201 (2007).

11. Y. Kawakami, S. Suzuki, A. Kaneta, M. Funato, A. Kikuchi, K. Kishino, Origin of high oscillator strength in green-emitting InGaN/GaN nanocolumns, Appl. Phys. Lett. 89, 163124 (2006).

12. H.-Y. Chen, H.-W. Lin, C.-H. Shen, S. Gwo, Structure and photoluminescence properties of epitaxially oriented $\mathrm{GaN}$ nanorods grown on $\mathrm{Si}(111)$ by plasma-assisted molecular-beam epitaxy, Appl. Phys. Lett. 89, 243105 (2006).

13. H.-S. Chen, D.-M. Yeh, Y.-C. Lu, C.-Y. Chen, C.-F. Huang, T.-Y. Tang, C.C. Yang, C.-S. $\mathrm{Wu}$, C.-D. Chen, Strain relaxation and quantum confinement in InGaN/GaN nanoposts, Nanotechnology 17, 1454 (2006).

14. S. Albert, A. Bengoechea-Encabo, P. Lefebvre, M. A. Sanchez-Garcia, E. Calleja, U. Jahn, A. Trampert, Emission control of InGaN nanocolumns grown by molecular-beam epitaxy on Si(111) substrates, Appl. Phys. Lett. 99, 131108 (2011).

15. H. W. Lin, Y. J. Lu, H. Y. Chen, H. M. Lee, and S. Gwo, InGaN/GaN nanorod array white light-emitting diode, Appl. Phys. Lett. 97, 073101, (2010).

16. A. L. Bavencove, G. Tourbot, J. Garcia, Y. Désières, P. Gilet, F. Levy, B. André, B. Gayral, B. Daudin and L. S. Dang, Submicrometre resolved optical characterization of green nanowire-based light emitting diodes, Nanotechnology 22, 345705, (2011).

17. W. Guo, M. Zhang, A. Banerjee and P. Bhattacharya, Catalyst-Free InGaN/GaN Nanowire Light Emitting Diodes Grown on (001) Silicon by Molecular Beam Epitaxy, Nano Letters 10, 9, 3355-3359 (2010).

18. K. Kishino, A. Kikuchi, H. Sekiguchi, and S. Ishizawa, InGaN/GaN nanocolumn LEDs emitting from blue to red, Proc. SPIE 6473, 64730T (2007).

19. H. Sekiguchi, K. Kishino, A. Kikuchi, Ti-mask Selective-Area Growth of GaN by RF-PlasmaAssisted Molecular-Beam Epitaxy for Fabricating Regularly Arranged InGaN/GaN Nanocolumns, Appl. Phys. Express 1, 124002 (2008).

20. K. Kishino, H. Sekiguchi, A. Kikuchi, Improved Ti-mask selective-area growth (SAG) by rfplasma-assisted molecular beam epitaxy demonstrating extremely uniform GaN nanocolumn arrays, J. Crystal Growth 311, 2063 (2009).

21. H. Sekiguchi, K. Kishino, A. Kikuchi, Emission color control from blue to red with nanocolumn diameter of $\mathrm{InGaN} / \mathrm{GaN}$ nanocolumn arrays grown on same substrate, Appl. Phys. Lett. 96, 231104 (2010). 
22. A. Bengoechea-Encabo, F. Barbagini, S. Fernandez-Garrido, J. Grandal, J. Ristic, M.A. Sanchez-Garcia, E. Calleja, U. Jahn, E. Luna, A. Trampert, Understanding the selective area growth of GaN nanocolumns by MBE using Ti nanomasks, J. Crystal Growth 325, 89 (2011).

23. B. Heying, R. Averbeck, L. F. Chen, E. Haus, H. Riechert, J.S. Speck, Control of GaN surface morphologies using plasma-assisted molecular beam epitaxy, J. Appl. Phys. 88, 1855 (2000).

24. S. Fernández-Garrido, J. Grandal, E. Calleja, M.A. Sánchez-Garcia, and D. López-Romero, A growth diagram for plasma-assisted molecular beam epitaxy of GaN nanocolumns on $\mathrm{Si}(111)$, J. Appl. Phys. 106, 126102 (2009).

25. C.S. Gallinat, G. Koblmüller, J.S. Brown, J.S. Speck, A growth diagram for plasma-assisted molecular beam epitaxy of In-face InN, J. Appl. Phys. 102, 064907 (2007).

26. Y.S. Park, S.-H. Lee, J.-E. Oh, C.-M. Park, T.-W. Kang, Self-assembled GaN nano-rods grown directly on (1 111$)$ Si substrates: Dependence on growth conditions, J. Cryst. Growth 282, 313 (2005).

27. R. Calarco, R.J. Meijers, R.K. Debnath, T. Stoica, E. Sutter, H. Lüth, Nucleation and Growth of GaN Nanowires on Si(111) Performed by Molecular Beam Epitaxy, Nano Letters 7, 2248 (2007).

28. R.K. Debnath, R. Meijers, T. Richter, T. Stoica, R. Calarco, H. Lüth, Mechanism of molecular beam epitaxy growth of GaN nanowires on Si(111), Appl. Phys. Lett. 90, 123117 (2007).

29. O. Landré, R. Songmuang, J. Renard, E. Bellet-Amalric, H. Renevier, and B. Daudin, Plasmaassisted molecular beam epitaxy growth of GaN nanowires using indium-enhanced diffusion, Appl. Phys. Lett. 93, 183109 (2008).

30. J. Ristic, E. Calleja, S. Fernández-Garrido, L. Cerutti, A. Trampert, U. Jahn, K.H. Ploog, On the mechanisms of spontaneous growth of III-nitride nanocolumns by plasma-assisted molecular beam epitaxy, J. Crystal Growth 310, 4035 (2008).

31. C.T. Foxon, S.V. Novikov, J.L. Hall, R.P. Campion, D. Cherns, I. Griffiths, S. Khongphetsak, A complementary geometric model for the growth of GaN nanocolumns prepared by plasmaassisted molecular beam epitaxy, J. Crystal Growth 311, 3423 (2009).

32. A. Trampert, J. Ristic, U. Jahn, E. Calleja, K.H. Ploog, TEM study of (Ga,Al)N nanocolumns and embedded GaN nanodiscs, Inst. Phys. Conf. Ser. 180, 167 (2003).

33. S. Fernández-Garrido, G. Koblmüller, E. Calleja, J.S. Speck, In situ GaN decomposition analysis by quadrupole mass spectrometry and reflection high-energy electron diffraction, $J$. Appl. Phys. 104, 033541 (2008).

34. J. Wu and W. Walukiewicz, Band gaps of InN and group III nitride alloys, Superlattices \& Microstructures 34, 63 (2003).

35. F. Barbagini, A. Bengoechea-Encabo, S. Albert, J. Martinez, M.A. Sanchez García, A. Trampert, E. Calleja, Critical aspects of substrate nanopatterning for the ordered growth of GaN nanocolumns, Nanoscale Research Letters 6, 632 (2011).

36. J.C. Hulteen and R.P. Van Duyne, Nanosphere Lithography: A materials general fabrication process for periodic particle array surfaces, J. Vac. Sci. Technol. A 13(3), 1553 (1995).

37. A. Bengoechea-Encabo, S. Albert, M.A. Sanchez-Garcia, L.L. López, S. Estradé, J.M. Rebled, F. Peiró, G. Nataf, P. de Mierry, J. Zuniga-Perez, E. Calleja, Selective area growth of $a$ - and $c$ plane GaN nanocolumns by molecular beam epitaxy using colloidal nanolithography, $J$. Crystal Growth 353, 1, 1-4 (2012).

38. M. Yoshizawa, A. Kikuchi, N. Fujita, K. Kushi, H. Sasamoto, K. Kishino, Self-organization of $\mathrm{GaN} / \mathrm{Al}_{0.18} \mathrm{Ga}_{0.82} \mathrm{~N}$ multi-layer nano-columns on $(0001) \mathrm{Al}_{2} \mathrm{O}_{3}$ by $\mathrm{RF}$ molecular beam epitaxy for fabricating GaN quantum disks, J. Crystal Growth, 189-190, 138 (1998).

39. H.-Y. Chen, H.-W. Lin, C.-H. Shen, S. Gwo, Structure and photoluminescence properties of epitaxially oriented GaN nanorods grown on $\mathrm{Si}(111)$ by plasma-assisted molecular-beam epitaxy, Appl. Phys. Lett. 89, 243105 (2006). 
E. Calleja et al.

40. P. Waltereit, O. Brandt, A. Trampert, H.T. Grahn, J. Menniger, M. Ramsteiner, M. Reiche, K.H. Ploog, Nitride semiconductors free of electrostatic fields for efficient white lightemitting diodes, Nature 406, 865 (2000).

41. V. Jindal and F. Shahedipour-Sandvik, Theoretical prediction of GaN nanostructure equilibrium and nonequilibrium shapes, J. Appl. Phys. 106, 083115 (2009). 